\title{
Análisis de terminología especializada para la traducción interdisciplinar de un webinario de temática médica: el caso de Rheumatoid Arthritis Pathophysiology (signs and symptoms)
}

\author{
Manuel Carmona Ruiz \\ Universidad de Córdoba \\ z92carum@uco.es
}

htpps://dx.doi.org.10.12795/futhark.2020.il5.02

Fecha de recepción: 5.09 .2020
Fecha de aceptación: 10.11 .2020

Resumen: La traducción interdisciplinar audiovisual-médica es un campo de la traducción poco explorado hasta el momento. Si bien es cierto que la traducción audiovisual es relativamente joven, la médica no lo es tanto. Es obvio que se necesitan ciertas habilidades y conocimientos para traducir un texto especializado: si es médico, conocimientos en traducción médica; si es jurídico, en traducción jurídica. Para su labor, el traductor necesitará, entre otros, analizar la terminología especializada presente en el texto. En el presente artículo se presenta un modelo de clasificación de términos que organiza la terminología y facilita su correcto análisis. Con este artículo se pretende, también, dar visibilidad a la traducción de webinarios, pues pueden aportar información y conocimientos muy beneficiosos para la sociedad.

Palabras clave: análisis terminológico, artritis reumatoide, traducción audiovisual, traducción interdisciplinar, traducción médica, webinario

Analysis of specialized Terminology for the interdisciplinary Translation of a Webinar on medical Subjects: the Case of Rheumatoid Arthritis Pathophysiology (signs and symptoms)

\begin{abstract}
Interdisciplinary audiovisual-scientific translation is an undeveloped field of translation so far. It is clear that medical translation enjoys a preeminent dedication of attention, effort, and resources due to it is worth large amounts of money and that it is really important in saving lives. For this purpose, the translator
\end{abstract}


needs to, among other, analyze the specialized terminology of the text. Therefore a term classification model has been developed in order to properly organize the terminology for it to be correctly analyzed. The aim of the work presented below is also to raise awareness to translation of webinars, as it is proved that they can provide very beneficial information and knowledge to society.

Keywords: audiovisual translation, interdisciplinary translation, rheumatoid arthritis, scientific translation, terminological analysis, webinar.

Sumario: Introducción. I. Motivación del estudio. 2. Fundamentación teórica. 3. Metodología. 4. Análisis terminológico. Conclusiones

\section{Introducción}

El término webinar es un anglicismo que hace referencia a «una presentación educativa en directo retransmitida por internet en la que los participantes pueden hacer preguntas y comentarios» (Merriam-Webster, 2020). Ante la diversidad de denominaciones, en este artículo voy a usar la versión híbrida webinario, siguiendo las pautas ofrecidas por la Fundación del Español Urgente (Fundéu): escritura en minúscula y en cursiva. Aunque el concepto se relaciona con una producción en directo, muchas organizaciones e instituciones, e incluso particulares, suben a la red sus webinarios para que se puedan ver de forma asíncrona (YouTube es un ejemplo claro). En mi Trabajo Fin de Máster (TFM) traduje el webinario de temática biosanitaria Rheumatoid Arthritis Pathophysiology (signs and symptoms) subido a YouTube. Para ello transcribí el audio y extraje y analicé la terminología especializada. Creo firmemente que un buen análisis de la terminología, así como su apropiada catalogación, conducen a una mejor traducción del texto.

Se parte de la concepción de la traducción como elemento que conecta culturas. Moreno y Rodríguez-Tapia (2015) consideran que la traducción surge cuando dos culturas, que hablan lenguas diferentes, entran en contacto y requieren de ayuda para comunicarse. No obstante, el traductor no puede dejarse notar, pues la traducción no debe dejar marcas visibles; un texto traducido debe dar una apariencia similar a la del original. Por ello es imperativo que la persona encargada de traducir un texto que presente dos modelos diferentes de traducción (médico y audiovisual, jurídico y técnico, médico y jurídico...) cuente con una formación interdisciplinar en ambos campos.

En un trabajo de finalización de estudios de Máster investigué sobre traducción interdisciplinar de las modalidades de traducción audiovisual y de traducción médica. Sin embargo, en el presente artículo pretendo centrarme en el 
análisis de varios ejemplos de la terminología especializada presente en el webinario que traduje. Para dicho análisis he desarrollado un modelo de clasificación basado en el sistema SKOS y en la Nomenclatura Internacional de la UNESCO para los campos de Ciencia y Tecnología. Puesto que en el trabajo aparecen más de 150 términos, he escogido los más representativos (bien por su dificultad o por su singularidad) para su exposición en este artículo.

\section{Motivación del estudio}

Esta investigación responde a una motivación personal con vistas a una futura posible aplicación tanto académica como laboral. Personalmente siento una singular predilección por dos especialidades de la traducción: la médica (traducción de informes médicos, prospectos de medicamentos, investigaciones...) y la audiovisual (series, películas, videojuegos, vídeos de plataformas de internet...). Este interés me lo han suscitado principalmente varias asignaturas del Grado en Traducción e Interpretación de la Universidad Pablo de Olavide y del Máster en Traducción Especializada de la Universidad de Córdoba: traducción especializada, introducción a la interpretación, traducción audiovisual, traducción biosanitaria, etc. Asimismo debo este interés a varias series de temática médica (The Good Doctor, Anatomía de Grey, The Resident, House...). Me parece fascinante traducir un tipo de contenido audiovisual que combina tanto el registro coloquial (pues no va dirigido a un público profesional) como el especializado (ya que contiene terminología, en algunos casos muy técnica, perteneciente al ámbito biosanitario) y que requiere una combinación de diversas destrezas traductoras.

Como he mencionado anteriormente, en este artículo pongo el foco sobre la terminología especializada. Terminología y traducción, sobre todo cuando se trata de textos especializados, van íntimamente ligadas, ya que si no se comprende todo el vocabulario específico de un texto es imposible traducirlo adecuadamente. Además, esta terminología debe organizarse y estructurarse para que se pueda consultar en el futuro si es necesario. Contar con corpus de terminología especializada puede ahorrar al traductor horas de trabajo de documentación, análisis y traducción. $Y$ ya se sabe que el tiempo es oro.

\section{Fundamentación teórica}

Ares (2006) realiza una aseveración sobre la serie House M. D. que resulta muy fácilmente extrapolable a la mayoría del resto de series médicas: «[House $M$. D.] presenta a los profesionales como todopoderosos y multidisciplinares, una situación irreal en la práctica diaria dada la superespecialización que hoy en día se exige a la profesión sanitaria» (Villalba, 2015: 2I). Todas las series sobre medicina incluyen una gran cantidad de términos especializados para otorgarle credibilidad y veracidad al argumento de la ficción. Villalba opina que esta «presencia de terminología específica en el guion objeto de traducción» añade una dimensión 
especializada a su carácter audiovisual (20I5: II). Asimismo considera que el traductor sigue enfrentándose a los problemas comunes que presenta un texto médico escrito, pues con frecuencia los guiones presentan un alto grado de especialización. No obstante, los guionistas de las series médicas deberían ser conscientes de que «la exactitud denominativa no es la regla general» en medicina (Balliu 200I: 35). Esto se refleja en el uso «de un lenguaje complejo y multidimensional, con diferentes variantes denominativas» (Villalba 2015: 12), definiéndose estas variantes como «la existencia de múltiples formas de designar un concepto a través de diferentes expresiones» (Tercedor, 2013: 8I).

A este fenómeno debe sumársele el de la supremacía del inglés como lingua franca de la «ciencia en general y la medicina en particular» (Villalba 2015: 12). EI problema, explica el autor, es que el tipo de inglés de los científicos actuales mezcla las reglas del inglés estándar con los rasgos propios de sus lenguas maternas. Ruiz indica que los científicos participan «actively in the creation of neologisms and technical terms» y considera que «they write in an individual and disorganized way» (2008: 234). Si se pone el foco en el plano lingüístico, Villalba expone que el español médico se ve afectado en todos sus niveles por la influencia del inglés. A nivel ortográfico, *amfetamina, *colorectal, *halucinación; a nivel léxico, confusión entre emergencia y urgencia o entre plaga y peste; y a nivel sintáctico, abuso de la voz pasiva, eliminación del artículo al comenzar una oración, etc. (Navarro: 200la). El principal inconveniente de que el inglés sea la lingua franca de la ciencia es que todas las publicaciones conducen a un «monolitismo intelectual» debido a que los autores «tienen a elegir [...] temas, conceptos y métodos de trabajo en función de los que se siguen [sic.] en los Estados Unidos» (Navarro, 200lb). Así, considera el autor, los principales problemas sanitarios de los Estados Unidos marcan la temática de los estudios nacionales e internacionales. Por último, Martínez (2008 citada en Villalba, 2015) considera que la supremacía cultural anglosajona en el lenguaje médico lleva a los autores extranjeros a adoptar las recurrentes metáforas y marcas comerciales del inglés, lo que conduce a un, a veces, inevitable uso del spanglish o lleva a traducciones literales incorrectas.

Hay que tener presente que la medicina en televisión implica un fenómeno social. La pequeña pantalla es un espejo de la sociedad; en ella se emite el contenido que la población reclama, contenidos en los que la gente se vea reflejada, que le ayude a evadirse de la realidad durante algunos minutos, etc. Por ello, el receptor de los productos audiovisuales especializados debe tenerse en cuenta a la hora de traducir estas producciones.

\section{Metodología}

Al ser el proyecto inicial un trabajo híbrido que combinaba investigación y práctica se estructuró en dos fases: 
Fase A: I. Se transcribió el texto origen en inglés a partir del vídeo colgado en YouTube; 2. Se extrajo la terminología especializada presente en el vídeo y se creó un glosario siguiendo un modelo de clasificación creado también para ese proyecto; y 3 . Se analizaron los términos, las dificultades de traducción de los mismos y las técnicas de traducción empleadas durante el proceso.

Fase B: 4. Se tradujo la transcripción al español; 5. Se detallaron los aspectos que se han tenido en cuenta a la hora de traducir; y 6 . Se obtuvieron unos resultados y unas conclusiones.

El fundamento de este artículo se halla en el punto 2. de la fase A: la etapa de análisis, en la que se extrajo y se analizó la terminología. Se creó un glosario y se analizaron tanto las dificultades como las técnicas de traducción empleadas. Respecto a la terminología, se extrajo el vocabulario especializado médico y se creó un modelo de clasificación para ordenarlo (el cual se explica con detalle en el siguiente apartado). Se tradujo el término y se definió en español. En lo que respecta al análisis, se explicaron las dificultades de traducción y se analizó qué técnica de traducción se usó $y$, en el caso de contar con varias opciones, por qué se optó por una y no por otra.

En el siguiente apartado se expone el análisis terminológico. Se presenta un modelo de clasificación específico creado para el TFM en el que se analiza el vocabulario, las dificultades de traducción y las técnicas utilizadas durante el proceso traslativo.

\section{Análisis terminológico}

Como mencioné al comienzo del artículo, analicé más de 150 términos en mi trabajo académico. Al ser una cantidad ingente de términos, he seleccionado los ejemplos más representativos de la investigación y del análisis. Para ordenar los términos desarrollé un modelo de clasificación basado en la Nomenclatura Internacional de la UNESCO para los campos de Ciencia y Tecnología y en el sistema SKOS.

La Nomenclatura Internacional de la UNESCO para los campos de Ciencia y Tecnología es una clasificación del conocimiento utilizada por las Administraciones del Estado para organizar los proyectos de investigación, tesis doctorales, etc. (Ruiz et al., 2014). Usando el sistema SKOS (Simple Knowledge Organization System), Pastor, Martínez e Iniesta (s. f.) recogen la clasificación que propone la UNESCO de categorías del conocimiento estructuradas en tres niveles de especialización: campos, disciplinas y subdisciplinas. Esta página de SKOS permite consultar la nomenclatura, tanto alfabética como jerárquicamente y así como descargar los archivos. 
La UNESCO codifica los campos con dos dígitos (ej. 32: Ciencias Médicas; 7I: Ética); las disciplinas con cuatro dígitos (ej. 3207: patología; 7103: ética de grupo); y las subdisciplinas con seis dígitos (ej. 3207.01: alergias; 7103.04: ética de la ciencia). A partir de este modelo organizativo, se desarrolla un modelo propio de clasificación de la terminología especializada que se ha extraído del vídeo objeto de estudio de este artículo. Se detalla a continuación:

Los términos se clasifican dentro de un campo de especialidad que se identifica con dos dígitos y de un campo temático que se identifica con cuatro dígitos (los dos primeros corresponden al campo de especialidad y los dos segundos al campo temático). Por último, se ordena con seis dígitos para identificarlos correctamente. Se muestra un ejemplo:

Ficha $n .^{\circ} 0$

\begin{tabular}{|l|l|l|}
\hline 10 & \multicolumn{2}{|l|}{ Endocrinología } \\
\hline 1030 & \multicolumn{2}{|l|}{ Sustancias } \\
1030.01 & EN: Glucocorticoid \\
\hline DEF: & $\begin{array}{l}\text { Hormona corticoesteroide segregada por la zona fasciculada de la } \\
\text { corteza suprarrenal. Su secreción la estimula la hormona } \\
\text { adenocorticoticotropa (ACTH) hipofisaria. Aumenta el nivel de } \\
\text { glucosa en sangre, moviliza el calcio óseo y se comporta como } \\
\text { antiinflamatorio. }\end{array}$ \\
\hline
\end{tabular}

Tabla I: Ejemplo de ficha terminológica

En el ejemplo se puede observar, de arriba a abajo, que se muestra el campo de especialidad (10. Endocrinología), el campo temático (1030. Sustancias), la clasificación bilingüe del término (1030.0I Glucocorticoid/Glucocorticoide) y, por último, se define el término.

Los campos de especialidad y campos temáticos, con sus respectivos códigos identificativos, se detallan en la siguiente tabla:

\begin{tabular}{|c|c|c|c|}
\hline Código & Campo de especialidad & Código & Campo temático \\
\hline 10 & Endocrinología & 10 & Enfermedades \\
\hline 20 & Cardiología & 20 & Procesos \\
\hline 21 & Hematología & \multicolumn{2}{|c|}{} \\
\hline 30 & Neurología & 30 & Sustancias \\
\hline 31 & Psicología & 31 & Organismos \\
\hline
\end{tabular}

Futhark I 5 (2020) 


\begin{tabular}{|c|c|c|c|}
\hline 40 & Inmunología & 40 & Síndromes \\
\hline 50 & Reumatología & 50 & Alteraciones \\
\hline 60 & Patología & 60 & Respuestas \\
\hline 61 & Hepatología & & \\
\hline 62 & Nefrología & & \\
\hline 63 & Neumología & & \\
\hline 70 & Anatomía & 70 & Pruebas \\
\hline 71 & Osteología & & \\
\hline 72 & Artrología & & \\
\hline 73 & Histología & & \\
\hline 74 & Miología & & \\
\hline 80 & Fisiología & 80 & Estructuras \\
\hline 90 & Microbiología & 90 & Materiales \\
\hline 91 & Genética & & \\
\hline 92 & Bacteriología & & \\
\hline
\end{tabular}

Tabla 2: Campos de especialidad y campos temáticos

Si un campo de especialidad o campo temático empiezan por el mismo dígito (por ejemplo: 20. Cardiología y 21 . Hepatología o 30. Sustancias y 31 . Organismos), significa que comparten características comunes. El que acaba en 0 es el campo genérico y el que acaba del I al 9 el campo específico.

Además, se ha hecho al mismo tiempo un estudio de las técnicas de traducción que se han utilizado para la traducción de la terminología. Dicho estudio se muestra debajo de cada tabla-ejemplo.

Ejemplo I.

Ficha $n .^{\circ} 3$

\begin{tabular}{|l|l|l|}
\hline 20 & \multicolumn{2}{|l|}{ Cardiología } \\
\hline 2010 & Enfermedades & EN: Myocardial infarction \\
2010.01 & ES: Infarto agudo de miocardio \\
\hline DEF: & $\begin{array}{l}\text { Síndrome coronario agudo que se caracteriza por una falta de } \\
\text { riego brusca (sufrimiento isquémico) a una parte del músculo del } \\
\text { corazón, producido por la obstrucción aguda y total de una de } \\
\text { las arterias que lo alimentan. }\end{array}$ \\
\hline
\end{tabular}


En español, el término correcto es «infarto agudo de miocardio» y no «infarto de miocardio», pues «agudo» significa «súbito, que alcanza de repente extrema intensidad». «Miocardio» procede del gr. myo "músculo" y del gr. kardi(a) "corazón", «músculo del corazón». Se puede elegir entre dos opciones diferentes de traducción: generalización o equivalente acuñado junto con ampliación lingüística. Coloquialmente se utiliza la generalización «infarto» para referirse al infarto agudo de miocardio, designación esta más académica y formal. Por otro lado, se puede utilizar este último término completo, «infarto agudo de miocardio», que incluye una pequeña ampliación («agudo»). En este caso se ha optado por el equivalente acuñado con ampliación lingüística para mantener el nivel del texto.

Ejemplo 2.

Ficha . $^{\circ} 5$

\begin{tabular}{|l|l|l|}
\hline 21 & Hematología \\
\hline 2110 & Enfermedades & EN: Plaque \\
2110.02 & ES: Ateroma \\
\hline DEF: & $\begin{array}{l}\text { Acumulación masiva de lípidos extracelulares que se observan } \\
\text { como masas lipídicas al microscopio óptico y suelen localizarse } \\
\text { en la capa musculoesquelética. Los ateromas estrechan el lumen } \\
\text { arterial y pueden provocar síntomas visibles como trombos o } \\
\text { hematomas. }\end{array}$ \\
\hline
\end{tabular}

El término «ateroma» proviene del lat. renacentista atherōma, que a su vez procede del gr. athēr- "papilla grumosa" y -ō-ma "resultado de un proceso". Se documentó en España por primera vez en 1570. Se trata, por tanto, de un equivalente acuñado de la versión inglesa.

Ejemplo 3.

Ficha $n .{ }^{\circ}$ II

\begin{tabular}{|l|l|l|}
\hline 21 & Hematología \\
\hline 2150 & Alteraciones \\
\hline 2150.01 & EN: Thrombocytosis \\
& ES: Trombocitosis \\
\hline DEF: & $\begin{array}{l}\text { Aumento anormal en el número de plaquetas. La forma benigna } \\
\text { o trombocitosis secundaria es asintomática y suele ser } \\
\text { consecutiva de un proceso inflamatorio, anemia hemolítica o }\end{array}$ \\
\hline
\end{tabular}


hemorragia, entre otros.

Es importante diferenciar entre «trombocitosis» y «trombocitemia». Cuando el aumento de plaquetas se debe a una condición oculta (infección, inflamación, etc.) se denomina «trombocitosis». Cuando la elevación plaquetaria se debe a una enfermedad de la sangre $o$ de la médula ósea se denomina «trombocitemia». En este caso, el vídeo hace referencia al aumento de plaquetas como consecuencia de la artritis reumatoide, por lo que se considera «trombocitosis», un préstamo naturalizado del alemán «Thrombozytose» que se convierte en español a «trombocitosis» para hacerlo más cercano a la fonología española.

Ejemplo 4: Ficha n. 13

\begin{tabular}{|l|l|l|}
\hline 30 & Neurología \\
\hline 3010 & Enfermedades & EN: Stroke \\
3010.01 & ES: Ictus \\
\hline DEF: & $\begin{array}{l}\text { Accidente cerebrovascular (ACV) súbito y violento resultado de } \\
\text { una interrupción repentina del riego sanguíneo en el cerebro, } \\
\text { por un coágulo, hemorragia o subida de tensión. }\end{array}$ \\
\hline
\end{tabular}

El término proviene del lat. ìctus "golpe”, “ataque”. En el latín clásico ya tenía un significado parecido al actual, «ataque súbito», aunque no era tan específico. En Inglaterra se utilizó en medicina para hablar de «pulso» en 1707 y uno siglo y medio más tarde, en I86I, se le dio el sentido actual en Francia. Se utiliza el equivalente acuñado ictus para su traducción.

\begin{tabular}{|c|c|c|}
\hline 40 & \multicolumn{2}{|l|}{ Inmunología } \\
\hline 4080 & \multicolumn{2}{|l|}{ Estructuras } \\
\hline \multirow{2}{*}{\multicolumn{2}{|c|}{4080.01}} & $\begin{array}{l}\text { EN: FC portion (Fragment crystallizable } \\
\text { portion) }\end{array}$ \\
\hline & & $\begin{array}{l}\text { ES: Fragmento RC (Fragmento de región } \\
\text { cristalizable) }\end{array}$ \\
\hline DEF: & \multicolumn{2}{|c|}{$\begin{array}{l}\text { Zona de la cola de un anticuerpo que interactúa con los } \\
\text { receptores de la superficie celular llamados receptores Fc y } \\
\text { algunas proteínas del sistema de complemento. Esta propiedad }\end{array}$} \\
\hline
\end{tabular}


permite que los anticuerpos activen el sistema inmunológico.

En inglés la terminología adecuada es «fragment crystallizable region ( $F C$ region)», por lo que la traducción al español es un calco léxico. No obstante, es difícil traducir la abreviatura porque se hace referencia a un fragmento de la región cristalizable, no a una región del fragmento cristalizable («Fc region»). El cluster inglés no tiene una traducción al español en el mismo orden, por lo que se ha optado por cambiar levemente la abreviatura, usando así la técnica de compensación.

Ejemplo 6: Ficha n. ${ }^{\circ} 47$

\begin{tabular}{|l|l|l|}
\hline 60 & Patología \\
\hline 6040 & Síndromes & EN: Swelling \\
& ES: Edema \\
\hline DEF: & $\begin{array}{l}\text { Hinchazón blanda de una parte del cuerpo, que cede a la presión } \\
\text { y es ocasionada por la serosidad infiltrada en el tejido celular. La } \\
\text { hinchazón, localizada o difusa, se debe al acúmulo del } \\
\text { componente extravascular del líquido extracelular de un } \\
\text { determinado órgano o tejido. }\end{array}$ \\
\hline
\end{tabular}

Este término tiene dos traducciones: «hinchazón» (coloquial) y «edema» (especializada). Se ha decidido usar el equivalente acuñado «edema» para seguir la línea de terminología especializada del texto origen.

\begin{tabular}{|c|c|c|}
\hline \multicolumn{3}{|c|}{ Ejemplo 7: Ficha n. ${ }^{\circ} 67$} \\
\hline 73 & \multicolumn{2}{|l|}{ Histología } \\
\hline 7380 & \multicolumn{2}{|l|}{ Estructuras } \\
\hline \multirow{2}{*}{\multicolumn{2}{|c|}{7380.01}} & EN: Joint tissue \\
\hline & & ES: Tejido articular \\
\hline DEF: & \multicolumn{2}{|c|}{$\begin{array}{l}\text { Agrupación ordenada de células de la articulación, de la misma } \\
\text { naturaleza, especializadas para desempeñar en conjunto una } \\
\text { determinada función en la articulación. }\end{array}$} \\
\hline
\end{tabular}

El sustantivo «tejido» procede del lat. tex(ere)- "tejer" y del español -do/da. La palabra texido se documentó en España por primera vez en 1218 y su uso 
anatómico en 1450. Respecto al adjetivo "articular» (articulāris en lat.), proviene del sustantivo «articulación» (articulātiōn(em) en lat.). Se trata, por consiguiente, de un equivalente acuñado.

Ejemplo 8: Ficha $n .^{\circ} 81$

\begin{tabular}{|l|l|l|}
\hline 90 & Microbiología \\
\hline 9030 & \multicolumn{2}{|l|}{ Sustancias } \\
\hline 9030.08 & EN: Fibroblast-like synoviocytes (FLS) \\
& ES: Fibroblastos sinoviales \\
\hline DEF: & $\begin{array}{l}\text { Componente celular residente más importante de la membrana } \\
\text { sinovial articular. Los FS desempeñan una función relevante en la } \\
\text { patogenia de la artritis reumatoide (AR). En la membrana } \\
\text { sinovial reumatoide, los FS aumentan en número (hiperplasia) y } \\
\text { muestran un fenotipo alterado que persiste en cultivo en } \\
\text { ausencia de estímulos externos. }\end{array}$ \\
\hline
\end{tabular}

Este término presenta dos posibles traducciones según la técnica de traducción que se aplique. La primera opción es traducir palabra por palabra: «Fibroblast» por «fibroblástico», «like» por «de tipo» y «synoviocytes» por «sinoviocitos», es decir, «sinoviocitos de tipo fibroblástico». La segunda opción es utilizar el equivalente acuñado «fibroblastos sinoviales». Izquierdo y Pablos (2013) adoptan ambas opciones. En este caso se escoge la segunda opción por ser más reducida $y$, por consiguiente, más sencilla de usar en un hipotético doblaje.

\section{Conclusiones}

Se considera que el subcampo de la traducción de webinarios es todavía un nicho de traducción que apenas se tiene en cuenta. Con este trabajo se pretende dar visibilidad a la necesidad de traducir este tipo de contenidos, pues pueden ser muy beneficiosos para la sociedad por su carácter divulgativo y por el interés que tiene la sociedad hacia temas médicos.

El Trabajo de Fin de Máster es un proyecto positivo para los estudiantes. Es innegable su utilidad en la formación como futuros traductores, pues supone un reto individual que requiere de todas las habilidades, técnicas, conocimientos y competencias que se adquieren a lo largo de su carrera universitaria $y$, posteriormente, a lo largo del Máster. El TFM es un pequeño ejemplo de lo que les depara a los estudiantes después de graduarse y realizar un máster. No obstante, la vida profesional no consiste en hacer proyectos similares al TFM. Después de este 
proyecto aún queda mucha formación por delante para poder introducirse $y$ mantenerse en un mercado laboral altamente hostil y competitivo en estos momentos.

La documentación es un proceso esencial a la hora de traducir un texto. Se debe buscar información sobre el tema tratado en el documento; se deben consultar textos paralelos; se debe extraer y traducir la terminología especializada de estos textos paralelos; y, por último, se debe extraer, buscar y traducir el vocabulario especializado del texto origen. El nuevo modelo de clasificación de terminología especializada puede ser de mucha utilidad para el traductor, pues le ahorra tiempo y esfuerzo ofreciéndole un glosario bien organizado de todos los términos médicos que se mencionan en el vídeo. Si este modelo de organización se nutre bien, el traductor podría encontrar clasificado en él la mayoría de los términos especializados a los que se va a enfrentar al traducir un documento biosanitario. En este caso en concreto, el modelo de clasificación se centra en la especialidad médica, lo que no resta que no se pueda aplicar a cualquier disciplina de la traducción: jurídica, técnica, turística, económica, etc.

Este análisis de terminología se enmarca en un Trabajo Fin de Máster que consistía en un proceso completo de traducción. Se identifican dos posibles líneas de trabajo futuras que podrían llevarse a cabo a partir del proyecto inicial. Por una parte, aunque se cumplieron los objetivo, el trabajo no se consideró inacabado, ya que podría continuarse. Para ello, sería posible utilizar la transcripción inglesa y la traducción española para subtitular el vídeo a ambos idiomas usando el programa Aegisubl y la traducción se podría también utilizar para doblar el vídeo al español. Para ello, se debería llevar a cabo un proceso de documentación sobre subtitulación y doblaje.

Por otra parte, el trabajo podría dar lugar a un proyecto de investigación de nivel superior; una tesis doctoral. La traducción interdisciplinar audiovisual-médica no está muy explorada en lo que respecta al campo de la investigación. El trabajo podría derivar en un proyecto de investigación de traducción interdisciplinar audiovisual-médica. Se ha observado también que, aunque existen glosarios o bases de datos terminológicas sobre términos médicos con su definición en español, como el diccionario médico de la Clínica Universidad de Navarra o el Dicciomed de la Universidad de Salamanca, se ha echado en falta la existencia de un diccionario bilingüe gratuito que recoja términos médicos en inglés y en español. Se podría crear, por consiguiente, una herramienta que cubra esta necesidad a partir del modelo de clasificación de terminología que se ha creado.

\section{Referencias bibliográficas}

\begin{tabular}{l}
${ }^{1}$ Consultar: http://www.aegisub.org/ \\
\hline Futhark I5 (2020) \\
19-32
\end{tabular}


3WC SEMANTIC WEB (2012). SKOS Simple Knowledge Organization System Home Page [en línea]. Recuperado el 11 de abril de 2020, de https://www.w3.org/2004/02/skos/.

ARES, A. (2006). «Libre elección de médico». En Villalba (2015). «La medicina en televisión: implicaciones para la traducción. El caso del doblaje de series sobre médicos». En SENDEBAR. Revista de Traducción e Interpretación. Universidad de Granada. N. ${ }^{\circ}$ 26, pp. 9-36. ISSN-e: 340-24I5.

BALLIU, C. (200I). «El peligro de la terminología en traducción médica». En Villalba (20I5). «La medicina en televisión: implicaciones para la traducción. El caso del doblaje de series sobre médicos». En SENDEBAR. Revista de Traducción e Interpretación. Universidad de Granada. N. ${ }^{\circ}$ 26, pp. 9-36. ISSN-e: 340-24I5.

CLÍNICA UNIVERSIDAD DE NAVARRA (s. f.). Diccionario médico. [En línea]. Recuperado el 1 de mayo de 2020, de https://www.cun.es/diccionariomedico.

FUNDACIÓN DEL ESPAÑOL URGENTE (2013). Seminario web, mejor que webinar [en línea]. Recuperado el 3 de marzo de 2020, de https://www.fundeu.es/recomendacion/seminario-web-webinario-mejorque-webinarl.

MARTÍNEZ, A. B. (2008). «Limitaciones y condicionantes de la práctica profesional de la traducción médica: aplicaciones a la combinación lingüística inglésespañol». En Villalba (2015). «La medicina en televisión: implicaciones para la traducción. El caso del doblaje de series sobre médicos». En SENDEBAR. Revista de Traducción e Interpretación. Universidad de Granada. N. ${ }^{\circ}$ 26, pP. 9. 36. ISSN-e: 2340-2415.

MERRIAM-WEBSTER (2020). Webinar [en línea]. Recuperado el 3 de marzo de 2020, de https://www.merriam-webster.com/dictionary/webinar.

MINISTERIO DE CIENCIA E INNOVACIÓN (2020). Nomenclatura Internacional de la UNESCO para los campos de Ciencia y Tecnología [en línea]. Recuperado el 7 de octubre de 2020, de https://www.ciencia.gob.es/portal/site/MICINN/menuitem.8ce192e94ba842 bea3bc811001432ea0/?vgnextoid=363ac9487fb02210VgnVCM1000001d04 140aRCRD\&vgnextchannel=28fb282978ea0210VgnVCM1000001034e20aR CRD

MORENO, M. ${ }^{a}$ C. y RODRÍGUEZ-TAPIA, S. (2015). «La situación de la traducción agroalimentaria en la investigación y la formación en España». En Skopos. Revista internacional de traducción e interpretación, N. ${ }^{\circ}$ 6, pp. 135-154. ISSN: 2255-3703. 
NAVARRO, F. A. (2001a). «El inglés. Idioma internacional de la medicina». En Villalba (2015). «La medicina en televisión: implicaciones para la traducción. El caso del doblaje de series sobre médicos». En SENDEBAR. Revista de Traducción e Interpretación. Universidad de Granada. N. ${ }^{\circ}$ 26, Pp. 9. 36. ISSN-e: 340-2415.

NAVARRO, F. A. (2001b). «La traducción médica ante el siglo XXI: tres retos para el lenguaje científico español». En Villalba (2015). «La medicina en televisión: implicaciones para la traducción. El caso del doblaje de series sobre médicos». En SENDEBAR. Revista de Traducción e Interpretación. Universidad de Granada. N. ${ }^{\circ}$ 26, pp. 9-36. ISSN-e: 340-2415.

PASTOR, J. A., MARTÍNEZ, F. J. e INIESTA, M. (s. f.). SKOS. Nomenclatura de Ciencia y Tecnología de la UNESCO. Recuperado el 11 de abril de 2020, de https://skos.um.es/unesco6/.

RUIZ, J. M., BAÑOS, M. J. y MARTÍNEZ, R. (2014). «Nomenclatura UNESCO: evolución, alcance y reutilización en clave ontológica para la descripción de perfiles científicos». En El profesional de la información (p. 384). Vol. 23(4), DOI: 10.3145/epi.2014.jul.06.

RUIZ, L. (2008). «English and Spanish Medical Languages: A Comparative Study from a Translation Point of View». En Villalba (2015). «La medicina en televisión: implicaciones para la traducción. El caso del doblaje de series sobre médicos». En SENDEBAR. Revista de Traducción e Interpretación. Universidad de Granada. N. ${ }^{\circ}$ 26, pp. 9-36. ISSN-e: 340-2415.

TERCEDOR, M. (2013). «Una perspectiva situada de la variación denominativa». En Villalba (2015). «La medicina en televisión: implicaciones para la traducción. El caso del doblaje de series sobre médicos». En SENDEBAR. Revista de Traducción e Interpretación. Universidad de Granada. N. ${ }^{\circ}$ 26, pp. 936. ISSN-e: 340-2415.

UNIVERSIDAD DE SALAMANCA (s. f.). Dicciomed. ISBN: 8478005722. [En línea]. Recuperado el 25 de abril de 2020, de https://dicciomed.usal.es/.

VILLALBA, R. (2015). «La medicina en televisión: implicaciones para la traducción. El caso del doblaje de series sobre médicos». En SENDEBAR. Revista de Traducción e Interpretación. Universidad de Granada. N. ${ }^{\circ}$ 26, Pp. 9-36. ISSNe: 2340-2415. 